\title{
40 million children have no access to the most basic healthcare services
}

In this News article by Rebecca Ghani (BMJ 2011;343:d4667 doi:10.1136/bmj.d4667) we omitted Dr Jennings' details. He is Michael Jennings, senior lecturer at the Department of

Development Studies, School of Oriental and African Studies, 